\title{
User's Search Behavior Graph for Aiding Personalized Web Search
}

\author{
Sendhilkumar .S $\mathrm{S}^{1}$ and Geetha $. \mathrm{T} . \mathrm{V}^{2}$ \\ 1,2 Department of Computer Science \& Engineering \\ College of Engineering, Anna University \\ Chennai - 600 025, India \\ \{thamaraikumar, tvgeedir\}@cs.annauniv.edu
}

\begin{abstract}
The www is a dynamic environment and it is difficult to capture the user preferences and interests without interfering with the normal activity of the user. Hence to improve user searching in the World Wide Web, we have proposed a personalized search system that supports user searches by learning about user preferences and by observing responses to prior search experiences aided by a new index called the User Conceptual Index (UCI). This paper models every user's search behavior as a User's Search Behavior (USB) Graph. The main focus of this paper is the analysis of the USB graph and the redesign of the UCI using the results arrived from the analysis.
\end{abstract}

Keywords: Personalization, Web Search, Web Information Retrieval, User Conceptual Index, User's Search Behavior Graph.

\section{Introduction}

The Web is a large collection of semi-structured and structured information sources and web users often suffer from information overload. General web search is performed predominantly through text queries to search engines. Because of the enormous size of the web, text alone is usually not selective enough to limit the number of query results to a manageable size. To alleviate this problem, personalization becomes a popular remedy to customize the Web environment for users and help them search their information need easily. Personalized search can be of two types: context oriented and individual oriented. By context oriented it means the interrelated conditions that occur within an activity. Individual oriented search means the totality of characteristics that distinguishes an individual. Context includes factors like the nature of information available, the information currently being examined, the applications in use, when, and so on. Individual oriented search encompasses elements like the user's goals, prior and tacit knowledge, past information seeking behaviors, among others.

The context of a search can be derived from the terms used in a search query. Likewise the individual search behaviors like, the links which the user clicks, the way in which the user moves from one page to the other and the actions like saving, printing or copy full or part of the page's content that were performed on the page viewed can be used to confirm the context of search derived form the search query. This insight has led S. Sendhilkumar and T. V. Geetha to design a search-aiding index called 
the UCI [2] that makes a search to be both context as well as individual oriented. The developed UCI utilizes the relationships between the search query and the pages visited by the user and thus it provides a ranking of the result pages based on the individual's context of search.

The main focus of this paper is towards the redesigning of the UCI based on the individual search behavior. A new graph called the User's Search Behavior (USB) graph has been discussed in this paper to model the user's browsing behavior. Also this paper explores the various analyses that can be performed on the USB graph and how these results can be of use for redesigning the UCI. Thus the redesigned UCI which provides both context as well as individual oriented search can improve the performance of search in the web by recommending pages that might be the direct answers to the user's information need.

\section{The User Conceptual Index (UCI) and Its Significance}

The proposed User conceptual index or User co-ordination index (UCI) is mathematically defined as in definition 1 .

Definition 1. Given a set of search queries $S Q$ and a set of relevant pages represented by their index words $I W$, User Conceptual Index can be defined as conceptual relation between the $S Q$ and the relevant pages, represented by a weighted function as given in (1).

$$
\mathrm{UCI}=f\left(W_{S K W} \rightarrow I W\right)
$$

where, $S K W$ is the search query, $I W$ is the index word(s) for a page and $W$ is the sum of the weight of any $S Q$ and their relevant $I W \mathrm{~s}$.

In equation $1 W$ is the sum of the weight of any $S Q$ and their relevant $I W(\mathrm{~s})$. This conceptual relation is an index for expanding the search query and for further page ranking modification. The proposed personalized search system identifies a matching $(S Q, I W)$ pair that has the highest UCI value and these pages will be recommended first to the users. These recommended combinations give the pages, which are direct answers to the user's information need.

While computing the average weight for $S Q$ factors like the frequency of terms used in the query, frequency of the query and the query usage time were considered. Similarly for computing the average weight for $I W s$ points like the page hits and the page view time were used.

Sample searches were conducted through Yahoo! Search engine to demonstrate the effectiveness of the proposed UCI based recommendation scheme [3].

The traditional performance evaluation measures for any IR based like precision and recall were used to evaluate [4] the search based on UCI. Recall measures how well a search system finds what the user wants and precision measures how well it weeds out the user don't wants and from the experimental results it was observed that there is a significant improvement of $9 \%$ in the overall efficiency of the UCI based search than the normal search [4].

Though it was a tedious process to compute the UCI values for all the result pages, the results proved the search to be more context oriented because the computed UCI 
contains information contributed directly by the user (i.e., the user queries) and also inferred by the system (like the hit count and page view times).

But still the UCI based search can be made more realistic and more relevant to the user's context of search by analyzing the individual's search behavior. From the user's browsing behavior useful patterns like user's interested pages, links that give the relationship between pages, pages that are indirect answers to the query, etc. can be derived. The following sections discuss about the link analyses technique and how it can be utilized to improve the UCI based search.

\section{Link Analyses and Its Usage in Tracing User Behavior}

Link analysis is a method that is used to determine what pages in the collection are important to users. Analysis of how pages link to each other in the web has led to significant improvements in web information retrieval [5]. Link analysis technology has been widely used to analyze the pages' importance, such as Hits [6] and Page Rank [7]. The goal of web search is to find all documents relevant for a user query in a collection of distributed web documents. Most search engines and related tools continue to ignore an essential part of the web - the links and continue making improvements in the information retrieval algorithms.

With the aim of utilizing the benefits of link analysis, this work represents the users click data (the links in a page which the user clicks while searching) as a graph. The user navigation through the web while searching for his/her information need can be represented as a graph, which can be used to analyze the user behavior, and hence the interests of the user from link analysis. The following sections describe how every user transaction and sessions as modeled mathematically for further analysis.

\subsection{Modeling User Search Behaviors}

From the client side user data collected two sets namely: the transaction set and the sessions set can be defined as in definition 2 and 3 .

Definition 2. Given a search query $S Q_{i}$ and the initial set of pages (technically represented by their IWs) retrieved by the search engine, a transaction $\mathrm{T}$ is defined as a set of pages visited by the user by clicking the links of the pages in the initial set of search results retrieved and is represented as $T=\left\{\mathrm{P}_{1}, \mathrm{P}_{2}, \cdots, \mathrm{P}_{\mathrm{n}}\right\}$, where $\mathrm{P}_{\mathrm{i}} \mathrm{C} . \mathrm{T}$ is represented as a set of $n$ page views, $P=\left\{p_{1}, p_{2}, \cdots, p_{n}\right\}$.

Page views are semantically meaningful entities which can be used for extracting useful information like users' interested web pages, relation between various pages, user interests, etc. Frequently occurring words in a web page are used as the IW.

Definition 3. Given a set of search queries $Q$, where $S Q_{i} \in Q$, a session is defined as a set of transactions, which can be represented as $S=\left\{T_{1}, T_{2}, \cdots, T_{m}\right\}$, where every $T_{i}$ represents one unique $S_{\mathrm{i}}$.

A session $S_{i}$ with a set of search queries $Q$ will directly represent the user's information need in that session. Every user search session can be modeled as a graph called the Search Flow Graph and the next session discusses about the modeling of the SFG. 


\subsection{The User's Search Behavior (USB) Graph and Link analysis}

A USB graph is a graph that represents a user's search transaction T showing how the user moves from one page to the other while searching for their information need, hence called as a flow graph. Mathematically a USB graph can be defined as given in definition 4.

Definition 4. A User's Search Behavior (USB) graph is defined as directed cyclic graph representing each user transaction $T=\left\{P_{1}, P_{2}, \cdots, P_{n}\right\}$, where $P_{i} \in T$ is represented as a set of $n$ pageviews, $P_{i}=\left\{p_{i 1}, p_{i 2}, \cdots, p_{i n}\right\}$.

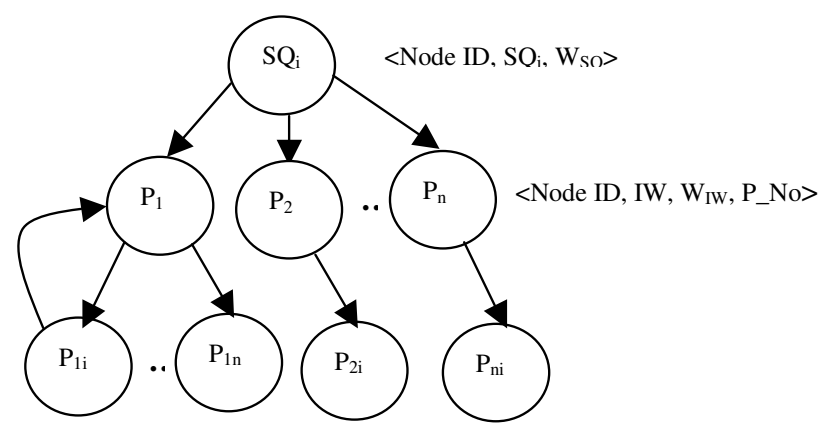

Fig. 1. User's Search Behavior (USB) graph

In figure 1 the root node represents the search query and the descendents of the root represents the pages viewed by the user. The backward arrow shows the user's movement from the current page to the previously viewed page. The root node in the SFG is represented using a 3-tuple $<$ Node ID, $\mathrm{SQi}, \mathrm{W}_{\mathrm{SQ}}>$, where $\mathrm{SQ}_{i}$ is the Search Query, $\mathrm{W}_{\mathrm{SQ}}$ is the average $\mathrm{SQ}_{\mathrm{i}}$ weight, Node ID comprises of Depth, Offset, where Depth represents the level and Offset is the node's position in the ith level and it is $\{0,0\}$ for the root node. All other nodes are represented using a 4-tuple <Node ID,

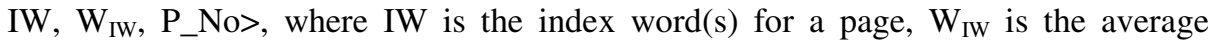
weight for the index words, $P \_$No is the $\{$Depth, Offset $\}$of parent node.

Table 2 presents the graph constructed in a tabular format for the sample query "IICAI 07" represented by the Node ID 00. The weight in the first row of table 2 represents the $\mathrm{W}_{\mathrm{SQ}}$ and all other weights indicate the $\mathrm{W}_{\mathrm{IW}}$. The weights are computed by taking an average of the time and frequency of usage of a SQ/IW through the various search sessions.

It can be seen from table 2 that the pages with node ID 20 and 30 represent the same page, but first the page with node id 20 is viewed by clicking the link from the page with node ID 10. So the parent page ID for the page 20 is 10 . And once again the same page was viewed by clicking a link present in another page (represented by the node ID 21) and so the parent page ID for page 30 is 21 . Also the table contains the ID of those pages that were viewed by clicking the back button. For example, the page with node ID 30 was visited by the user well before by 
Table 2. Sample USB graph constructed for the query IICAI 07 in tabular format

\begin{tabular}{|l|l|l|c|c|c|}
\hline $\begin{array}{l}\text { Node } \\
\text { ID } \\
\text { <Depth } \\
\text {, Off- } \\
\text { set> }\end{array}$ & Visited Page's URL & $\begin{array}{l}\text { Index Words } \\
\text { (IW) }\end{array}$ & $\begin{array}{c}\text { Weights } \\
\left(\mathrm{W}_{\text {SQ }}\right. \\
\left.\mathrm{W}_{\text {IW }}\right)\end{array}$ & $\begin{array}{c}\text { Parent } \\
\text { Page } \\
\text { ID }\end{array}$ & $\begin{array}{c}\text { Back } \\
\text { Track } \\
\text { ID }\end{array}$ \\
\hline 00 & www.google.com & & 0.498705 & - & \\
\hline 10 & http://www.iiconference.org/ & $\begin{array}{l}\{\text { conference, } \\
\mathrm{AI}\}\end{array}$ & 0.780034 & 00 & \\
\hline 11 & $\begin{array}{l}\text { http://www.iiconference.org/iicai07 } \\
\text { / }\end{array}$ & $\{$ index $\}$ & 0.008243 & 00 & \\
\hline 12 & $\begin{array}{l}\text { http://www.cl.cam.ac.uk/ jac22/cfp } \\
\text { /msg00248.html }\end{array}$ & $\{$ iicai-07\} & 0.012534 & 00 & \\
\hline 20 & $\begin{array}{l}\text { http://www.iiconference.org/session } \\
\text { s.html }\end{array}$ & $\{\mathrm{AI}\}$ & 0.267801 & 10 & 10 \\
\hline 21 & $\begin{array}{l}\text { http://www.iiconference.org/dates.h } \\
\text { tml }\end{array}$ & $\{2007\}$ & 0.253788 & 10 & 10 \\
\hline 22 & $\begin{array}{l}\text { http://www.iiconference.org/submis } \\
\text { sion.html }\end{array}$ & $\{$ paper $\}$ & 0.076756 & 10 & 10 \\
\hline 30 & $\begin{array}{l}\text { http://www.iiconference.org/session } \\
\text { s.html }\end{array}$ & $\{\mathrm{AI}\}$ & 0.006329 & 21 & 21 \\
\hline 31 & $\begin{array}{l}\text { http://www.iiconference.org/iicai07 } \\
\text { /swir.html }\end{array}$ & $\begin{array}{l}\{\text { semantic, } \\
\text { web }\end{array}$ & 0.849056 & 20 & 20 \\
\hline
\end{tabular}

clicking the link available in page 10. But the user on seeing the same page by clicking a link from page 21 goes back to the previous page from where he/she selected the link. This is indicated by the back track ID in table 2 . Such back tracking will help us to identify the pages that provide the user with useful and nonredundant links. Using this detail limited set of useful pages without redundant links can be recommended to the user.

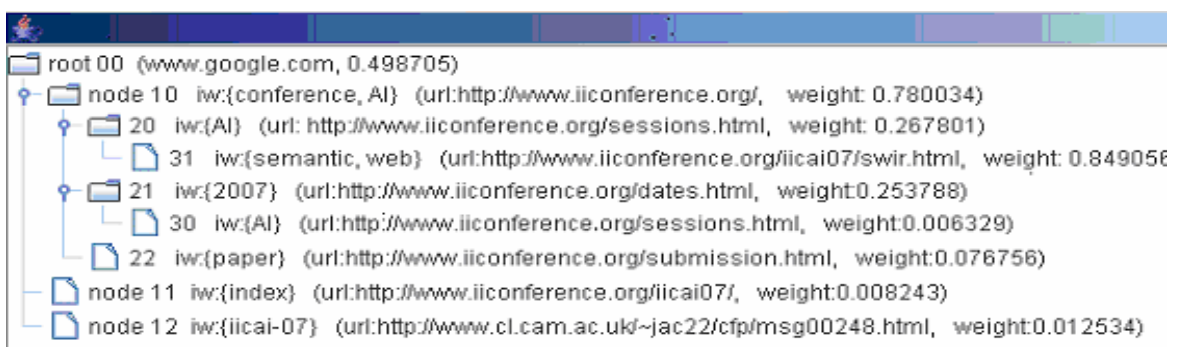

Fig. 2. USB Graph constructed for the data in table 2

The graph constructed for the data given in table 2 is shown in figure 2 . In figure 2 the node ID, URL, index words and the index word weight, represents each node. Every node in the graph is an expandable node and by clicking the expandable button we can view the links visited from a specific page.

Thus the main advantage of the USB graph is that it includes only those pages viewed by the user and maintains the order in which the pages were viewed. Hence only a reduced set of pages can be used for the computation of the UCI. Using the 
page weight and view time it can be concluded whether the transaction is useful for the user's search or not.

\section{USB Graph Analyses and Modification of the UCI}

The USB graph thus constructed includes both the spatial features as well as the temporal feature. The spatial temporal features like the hits and the page view time respectively are considered for index weight calculation. Hence Link analysis can give significant contributions to web page retrieval from search engines, to web community discovery, and to the measurement of web page influence. It can help to rank results and find high-quality index/hub/link pages that contain links to the best sites on the user's topic of interest.

Also from the sub-trees of the USB graph, page view levels (the depth to which a user might move to find their information needs) can be identified and such information depth levels can be used as an indication for the user to stop searching further. Also from the experiments conducted and from the USB graph it was found that the maximum level of depth that was traversed by the user was 12 and it was for the query "News NDTV". For other queries like "NLP", "java", "data mining" and "Artificial Intelligence Conferences" the maximum depth was 9. For the queries "IICAI 07", "deadlock" and "Anna University exam results" the depth of search was 3(from table 2) and 2 respectively.

\subsection{Redesign of the UCI}

Each page is assigned a weight based on its relevance to the search word, where the relevancy is to what extent the search keyword $S K W$ matches with the index/feature words $I W$ of the pages returned as a result of the user search. The page weight $w$ is a defined as a function $\quad f\left(W_{S K W} \rightarrow I W\right)$ called the search keyword - relevant page weight function [10]. The average weight for each transaction can be calculated by using the formula:

$$
\mathrm{W}_{\mathrm{ti}}=\Sigma \mathrm{W}_{\mathrm{Pi}}+\left(-\Sigma \mathrm{W}_{\mathrm{Pj}}\right)
$$

where, $\mathrm{W}_{\mathrm{ti}}$ is the $\mathrm{i}^{\text {th }}$ transaction' $\mathrm{s}$ weight, $\mathrm{W}_{\mathrm{Pi}}$ is the weight of $\mathrm{i}^{\text {th }}$ page $P_{\mathrm{i}}$. The negative sign in the second half of the above formula signifies back tracking. Every time the user backtracks from the $\mathrm{j}^{\text {th }}$ page to the $\mathrm{i}^{\text {th }}$ page we increase the weight of the $\mathrm{i}^{\text {th }}$ page and reduce weight of the $\mathrm{j}^{\text {th }}$ page.

In any user transaction if the user has already visited a page, say $P 1$ and during the process of searching the user finds that the same page $P l$ getting referred or linked in another page, then we consider that the page $P 1$ as an important/relevant page for the given search keyword $S K W$ and hence the weight for the page $P$ i can be increased. Now for each search keyword we have n number of transactions and the weights of respective transactions $\mathrm{W}_{\mathrm{ti}}$ can be used in identifying the pages of users interest. In the previous work of Sendhilkumar .S and Geetha .T.V [10] search keyword-relevant page weight function $f\left(W_{S K W_{\mathrm{i}} \rightarrow P \mathrm{i}}\right)$ and the page relation weight function $f\left(W_{P \mathrm{i}} \rightarrow P_{\mathrm{j}}\right)$ from the data collected was calculated. The page relation weight is assigned based on the relevancy between any two pages, i.e., to what extent the index/feature words $I W_{\mathrm{i}}$ 
of the ith page $P_{\mathrm{i}}$ matches with that of the index/feature words $I W_{\mathrm{j}}$ of the jth page $P_{\mathrm{j}}$. These two weighted functions can be used to represent user's current task.

From the transaction weights $W_{\mathrm{ti}}$ user-interested transactions can be identified and with the aid of such interest-oriented transactions filtering of unwanted transactions can be done and thus user's information needs can be reached more easily. These user-interested transactions will contain pages relevant to the given user's search keyword $S K W$. Therefore from the link analysis can be identified the most relevant pages to a particular $S K W$. The percentage of relevancy between the pages and the search keyword can be confirmed from the user's current search behavior, which is represented as a graph in our work. Hence we increase the weight of a search keyword and its relevant page in the search keyword-relevant page weight function $f(W$ $S K W_{\mathrm{i}} \rightarrow P_{\mathrm{i}}$ ) by adding the new search keyword-relevant page weight derived from the transaction link analysis to the previously calculated search keyword-relevant page weight. Similarly the related pages can be identified from the user-interested transactions and hence $\mathrm{i}$ the weight of related pages can be increased in the page relation weight function $f\left(W_{P \mathrm{i}} \rightarrow P_{\mathrm{j}}\right)$. Thus the two weighted functions: the search keywordrelevant page weight function and the page relation weight function get modified from the results of transaction link analysis.

Thus the final weight for a search keyword $\mathrm{w}\left(\mathrm{SKW}_{\mathrm{i}}\right)$ for which a user is searching in a session is given in (3).

$$
\mathrm{W}\left(\mathrm{SKW}_{\mathrm{i}}\right)=\Sigma\left(W_{S K W \mathrm{i}} \rightarrow P_{\mathrm{i}}\right) \cdot \Sigma\left(W_{P \mathrm{i}} \rightarrow P_{\mathrm{j}}\right)+\mathrm{W}_{\mathrm{Ti}}
$$

Thus a new modified weight is calculated for the search keywords by taking into consideration the user's current search behavior.

Finally the new search keyword weight $\mathbf{w}\left(\mathbf{S K} \mathbf{W}_{\mathbf{i}}\right)$ can be added to the previously calculated UCI as in (4).

$$
\mathrm{UCI}=f\left(W_{S K W_{\mathrm{i}} \rightarrow P \mathrm{i}}\right)+\mathbf{w}\left(\mathbf{S K W}_{\mathbf{i}}\right)
$$

This newly modified UCI weight that takes care of both current and previous interests of users can be used in the recommendation process such that if the weight is below a threshold then those combinations can be rejected and others can be recommended. These recommended combinations give the pages, which are direct answers to the user's information need. Filtering systems determine which documents in the result sets are relevant and which are not. Good filters remove many non-relevant documents and preserve the relevant ones in the results set. Now these filtered pages can be re-ranked using any ranking algorithm and presented to the user.

\section{Conclusions}

In summary this paper has incorporated a relation between the web content and the search key words by means of a new index called the modified User Conceptual Index. This paper models every user search behavior as a User's Search Behavior (USB) graph. The modified User Conceptual Index takes into account about the user's interested transaction and sessions that contain relevant pages, which match their 
information need. This refined User Conceptual Index can be used in any recommendation process at any stage of the search. We are still in the process of refining the UCI so that it also takes into account the user's shift in interests and the rate of decay of user's interest on some concepts. Further clustering techniques have been planned for clustering the user groups from the data collected by the proposed agent program and to include dimensionality reduction techniques to make use of a reduced set of data for future analysis.

\section{References}

[1] Page, L., Brin, S., Motwani, R., Winograd, T.: The PageRank citation ranking: Bringing order to the Web. Technical report, Stanford University Database Group (1998), http://citeseer.nj.nec.com/368196.html

[2] Sendhilkumar, S., Geetha, T.V.: Web Search using personalized user conceptual index. In: Proceedings of 2nd Indian Interantional Conference in Artificial Intelligence, pp. 17191728 (December 2005)

[3] Sendhilkumar, S., Geetha, T.V.: Personalized Web Search Using Enhanced Probabilistic User Conceptual Index. Special Issue of the Journal of Intelligent systems (in Press)

[4] Sendhilkumar, S., Geetha, T.V.: An Evaluation of Personalized Web Search for an Individual User. In: the International conference in Artificial Intelligence and Pattern Recognization (AIPR 2007), Orlando, USA, pp. 484-490 (July 2007)

[5] Henzinger, M.: Link Analysis in Web Information Retrieval. IEEE Data Engineering 23(3), 3-8 (2000)

[6] Kleinberg, J.M.: Authoritative sources in a hyperlinked environment. Journal of the ACM 46(5), 604-632 (1999)

[7] Brin S., Page, L.: The anatomy of a large-scale hypertextual web search engine. In: Proc. of WWW 2007 Brisbane, Australia, pp. 107-117 (April 1998) 